\title{
Patient Safety in Pediatrics
}

\author{
Sara Albolino, Marco De Luca, \\ and Antonino Morabito
}

\section{Learning Objectives/Questions Covered in the Chapter}

- What are the most common adverse events in pediatrics?

- Which are the approach and solutions to prevent harm?

- What are the most critical issues for quality and safety in the process of care of children?

- Which are the most effective practices and strategies for implementing them?

\subsection{Epidemiology of Adverse Events in Pediatrics: Some Numbers and Some Reflections}

Since the publication of the 1999 IOM report "To Err Is Human: Building a Safer Health System,"

\footnotetext{
S. Albolino $(\bowtie)$

Centre for Clinical Risk Management and Patient Safety, WHO Collaborating Centre in Human Factors and Communication for the Delivery of Safe and Quality Care, Tuscany Region, Florence, Italy e-mail: albolinos@aou-careggi.toscana.it

\section{De Luca}

Simulation and Risk Management, A. Meyer Children Hospital, Florence, Italy

e-mail: marco.deluca@meyer.it

\author{
A. Morabito \\ Pediatric Surgery, Meyer Children's Hospital, \\ University of Florence, Florence, Italy \\ e-mail: antonino.morabito@unifi.it
}

much has been learned about pediatric patient safety. However, adverse events still affect onethird of all hospitalized children [1]. The main areas of adverse events are hospital-acquired infections, intravenous line complications, surgical complications, and medication errors [2].

The area of medication errors that has been considered the major priority by WHO through the campaign "medication without harm" [3] for children is even more critical because of childhood development, demographics, dependency on parents and other care providers, and the different epidemiology of medical conditions [4].

In a study conducted in 2 pediatric hospitals, on 1120 admission, including more than 10,000 orders, there were 616 medication errors $(5.7 \%)$ [5]. In recent years the transition to digital healthcare has been identified as a significant element that concur to medication errors, in fact the electronic clinical records can affect the safety of prescription until they are modified with customized decision support, such as weight-based and body surface area-based dosing [2].

Concerning the most critical settings for quality and safety, a study published in 2012, conducted in Canada in 8 teaching hospitals and 14 local healthcare agencies underlined that the $79 \%$ of adverse events in children happened in intensive care unit, of which more than $40 \%$ are preventable and the incidence on admissions is of $6.5 \%$ [6]. These results are similar to the studies conducted in the adult population. 
Other studies show other types of errors with total rates as high as 40 harms per 100 patients. Events reported include accidental extubation, pressure ulcers, patient misidentification, delays in diagnosis, intravenous infiltrates, and other adverse events attributed to systemic causes like communication, training, and systems failures [7].

Concerning the ambulatory setting, a recent study [8] revealed that the largest group of errors was attributed to medical treatment (37\%). Other errors included patient identification (22\%), preventive care (15\%), diagnostic testing (13\%), and patient communication ( $8 \%$ ).

In a meta-analysis conducted in 2018 , with the analysis of 388 primary studies related to interventions to improve patient safety, the most common critical issues to address were medication (189 studies, 48.7\%) and general medical (81 studies, $20.9 \%$ errors) whereas the $53.1 \%$ (206 studies) addressed healthcare systems and technologies [9].

From these and other evidences, we can affirm that over the last 20 years many efforts and advancements have been done to improve patient safety, even in pediatrics, but the trend doesn't seem to have changed, as the complexity of the healthcare systems and their dynamicity is a big challenge for improving quality and safety of care. Also the centrality of the human being, the continuous interaction of clinicians and patients with the other components of the system, makes risks and errors in healthcare an unavoidable fact.

Error is part of normal human behavior as highlighted by James Reason "Human error is both universal and inevitable-human fallibility can be moderated but it can never be eliminated [10]. However, when errors have significant consequences or occur in high-risk industries, they become of paramount importance (ibidem)". Reason wrote that "Errors are consequences not causes, they have a history" and that only by understanding the history of the circumstances can there be progress to limit the chances of a recurrence (ibidem).

As a result of high-profile errors which have caused many deaths, industries like aviation, space travel, military, nuclear, and oil rigs have spent the last decades investing in the understanding, identification, and error prevention training in their respective industries [11].

In order to understand and prevent that errors result in adverse events, it is important to adopt a patient safety approach with some key elements: awareness of the epidemiology of adverse events and establishment of methods for risk identification; integration of improvement science principles and techniques into daily work; and creation, customization, and application of evidence- and context-based patient-safety solutions. Each of these key elements can be incorporated into pediatric patient safety risk assessment and solution development [12].

Some of the most common and severe types of adverse events, also in pediatrics, are the ones that happen in surgery. All surgery involves risk and potential complications - both known and unexpected. Medical errors may be decreased if there are clear standards of care described and adhered to; however it is clear that no two surgeries are identical; therefore every surgical error (and the underlying cause of the error) has the potential to be unique [13].

In high-reliability organizations variability is a constant and the focus is on minimizing that variability and its effects, this strategy in highhazard industries have contributed in a significant decrease of severe adverse events. But in the specific setting of healthcare, with the high complexity of medical care there are usually more difficulties in creating a culture of safety. In this case, as underlined by Pascale Carayon in her SEIPS model [14], the science of human factors (the focus on how people interact with each other and their environment) provides healthcare professionals with an important view that can give them the resilience to avoid adverse events. An optimal culture of safety focuses on human fallibility by concentrating on the conditions under which people work and on building defenses to prevent adverse events or mitigate their effects.

Going back to the surgical adverse events in pediatrics, not all surgical errors constitute medical malpractice and not all errors lead to adverse 
events. Studies indicate that nearly $75 \%$ of surgical errors occur during surgery, with the remaining occurring either during pre- or postoperative care. There are well-recognized factors for surgical errors [15-18]:

- Environment-Factors in the organizational and management structure/culture may impact on the individual surgeon performance from lack of a safety culture, lack of effective leadership and workplace communication procedures.

- Lack of Surgical Competency-be that lack of the appropriate individual technical skills or technique, poor decision-making, poor teamwork.

- Insufficient Preoperative Planning-It is critical that a surgeon be well prepared for surgery with a full knowledge of the individual patient to be operated on the intended techniques. The preparation of the patient is also important including a robust preoperative workup and multidisciplinary discussions if necessary of any known or anticipated issues/complications. Preoperatively the surgeon should try and forward plan and anticipate complications that have a potential to occur. The wider theater team needs to also have had the opportunity for forward planning, ensuring the correct skill mix is present, the correct equipment available with personnel familiar with the operative procedures to be undertaken and equipment to be used.

- Inappropriate workplace conduct-Either professionally by utilizing improper surgical techniques and employing improper surgical haste or "shortcuts" or personal by, e.g., disruptive behavior and poor leadership.

- Poor Communication-This can be as an individual or within the team. Poor communication can occur prior to the operation, e.g., marking the wrong site for surgery, miscommunication of the procedure to occur, and lack of forward communication about surgical equipment required. To improve communication prior to the start of the theater list Team Briefing is now employed.
- Fatigue, personal stresses, drugs, and alcohol can all impair decision-making and technical performance.

- Patient factors may make the surgery more complex such as the ASA grade, age, BMI, and surgical pathology (e.g., previous surgery) and these can all influence performance and postoperative recovery.

Surgical competency involves a combination of good decision-making (preoperatively, operatively and postoperatively), team performance and communication with all colleagues and the appropriate technical skills. These skills coupled with a high patient volume operating rate tend to achieve a reduced patient mortality and morbidity $[19,20]$. There are three important "red flag" times to check the correct patients/procedures/ skill and equipment mix-before induction of anesthesia, before skin incision, and before the patient leaves the theater suite. During the procedure the lead surgeon should be constantly reevaluating the ongoing surgical progress with salient communications; they should be reappraising the clinical and theater setting, and constantly re-evaluating the patient's care and the conduct of the operation. Protocols have been introduced to reduce surgical error (e.g., safer surgery checklists) [21]. However, the occurrence of surgical error is part of a multifaceted phenomenon and protocol use is only part of the overall solution. A cascade of errors from varying aetiologies, with different controlling factors, can/may culminate into a catastrophe or adverse event [22].

Practicing surgery and medicine in the twentyfirst century will embrace new medications, technologies, equipment, operations, etc. which aim to improve the treatment and care of patients. However the focus must also remain on evaluating and minimizing the impact of adverse events in the healthcare environment to provide a sustained, high level of surgical care.

Pediatric healthcare providers in all practice environments can benefit from having awareness of the need for understanding patient safety starting from a systemic and human factor approach. 
Knowing the patient safety concepts and language can help pediatricians to adopt the best practices and attend risks that are unique to children, and leading efforts to reduce avoidable harm for their patients [2].

\subsection{The Importance of Understanding the Context for Patient Safety Practices for Pediatrics}

Epidemiological data and evidences from the literature are few for patient safety in pediatrics and there is a need for comparing experiences and applied solutions in different contexts. At the European level some recommendations have been defined and the need for applying specific solutions and patient safety practices has been underlined. We have now a list of evidence-based patient safety practices at the international level. These practices need to be adapted by trials in order to be useful also for the pediatric settings. The patient safety manifesto by the American Academy of Pediatrics whose last version was published in 2019 underlines the importance to evaluate the patient's specific characteristics every time that a safety intervention for children is planned [2].

In fact the analysis of patient safety practices in pediatrics literature underlines that there is no list of interventions strongly recommended [23], in contrast with the adult context where some priority interventions have been identified [24], while there are several groups of practices that can be adopted according to the specificity of the pediatric setting and issue you have to address. The concern with a compiled unique list, say the authors, is to give priority to practices which are less expensive or more adapted to the hospital setting. Especially in pediatrics it is instead important to consider the complexity of the care, the inestimable value of saving a life and the fragility of the patients. Besides that, it is important to consider that in general there is a need for moving from simple to complex interventions which include not just the application of single actions but the identification of more advanced model for evaluating complexity and promote a system change.

\subsubsection{Simulation as a Key Factor for Implementation of Solutions for Safety in Pediatrics}

One of the most effective methodologies for evaluating the complexity, developing technical and nontechnical skills, and testing a system change is simulation. Pediatrics is among the disciplines that benefits most from the introduction of simulation, the health personnel who take care of the health of children have the possibility of exercising and improving their skills, without risk and without the emotional stress of damaging their little patients [25]. This is an extraordinary context to experiment new solutions for safer care.

The pediatric patient has unique characteristics, both from a physiopathological and psychobehavioral point of view. "The child is not a small adult" and this assumption also applies to simulation. The pathology of the critically ill pediatric patient is often a rare but high-risk pathology. This involves the use of medical devices and supplies specific for the child, with calibers and dosages that vary with the weight and age of the patient. All this adds complexity and risks to an already complex situation.

However, the simulation is an added value not only for training on technical skills. It is now clear that many errors in the medical field are consequent to problems related to the organization and not to the inexperience of the professionals or to negligence or incorrect individual conduct. Research has also shown that through the development of a process-centered model in the health system, it is possible to reduce adverse events and improve the quality and safety of care. Since 2000, the Institute of Medicine [26] mentions simulation as a key strategy for improving patient safety.

It is also important to mention that in order to apply solutions for improving quality and safety 
of health in an effective way, the simulation is a key element. Especially simulation on site had a significant diffusion in relation to this purpose. In fact simulation on site is less expensive and can be performed in the everday working environment, so that a major number of healthcare operators can participate and experience the advantage of testing a solution. With the application of a structured debriefing, it is possible to evaluate the positive and negative aspects of the designed solution and tailor it for a better compliance to the specific setting.

The use of simulation offers undoubtable advantages, among the most relevant are:

- Eliminates all the problems related to fear, embarrassment, possible legal implications that can arise during a real event.

- Allows, once the causes of an adverse event or near-miss has been identified, to define corrective measures which, if applied in a subsequent planned simulation, offer the possibility of verifying their feasibility and effectiveness in the field.

- Allows to evaluate the clinical risk of processes that in reality are only exceptionally implemented, but which can be tested several times as part of a simulation project.

- In addition to the ethical and moral implications of providing the highest quality in care, there are important financial reasons for introducing simulation more frequently within health systems. Whereas it is now clear that adverse events significantly increase the cost of treatments. It is likely that decision makers will be more receptive in incorporating the simulation within healthcare organizations, if the value of this training, also in terms of return on investments, can be demonstrated tangibly.

In the next paragraph we present a clinical case, based on a true reported event, that is also used, in a large network of healthcare trusts for training by simulation pediatricians in the emergency area (Simpnet-Tuscany regional network for pediatric simulation).

\subsubsection{Clinical Case: Safe Care in Pediatric Emergency}

\subsubsection{9.15 pm}

Anna arrives in PS, transported by an ambulance with a doctor on board, Anna is a 3-year-old patient with perinatal suffering quadriparesis. Anna has been suffering from high fever gastroenteritis for the last 2 days. In the past $12 \mathrm{~h}$, she has had numerous episodes of vomiting and diarrhea. For the last $4 \mathrm{~h}$, she has been more sleepy and less interactive with her mother, who worried and called the emergency service, when after the last episode of vomiting, the daughter did not respond to verbal stimuli.

These are the vital signs recorded during ambulance transportation:

- RF 36/min

- $\mathrm{satO}_{2}$ not detectable due to the presence of peripheral vasoconstriction

- CF $150 / \mathrm{min}$

- $\mathrm{T} 37.6{ }^{\circ} \mathrm{C}$

- AP 72-41

When the ambulance arrives at the ER, Anna is transferred directly to the red code room (resuscitation room). Giulia, the triage nurse, asks the mother to stay at the triage with her for collecting the personal and clinical data of the patient. The door of the red code room closes and Anna's mom will never enter that room during the resuscitation phases. She will wait sitting on a chair just outside the door of the red code room.

\section{Critical Issues}

International literature and guidelines agree in making the family members of pediatric patients to assist to the resuscitation phases, if they wish, and in any case to encourage their presence. This presence should not be perceived as invasive for the resuscitation team. Evidences show that the parents' presence benefits the child and allows the family members to have a realistic view of the resuscitation attempts and of the expertise of the operators. In addition, it helps the parents in 
developing greater adaptability if the patient dies and in experiencing a better mourning process.

\subsubsection{9.18 pm}

In the room there are:

- Mario-Pediatrician with 6-year experience in the ER

- Sara-Pediatrician with a temporary contract for 3 months in the ER

- Lucia-Expert nurse in the ER

- Claudio-nurse transferred to the ER for 6 months from a geriatric ward

- Paolo-doctor working at the emergency territorial service

- Cesare-volunteer of the emergency territorial service

Mario asks Sara to get Anna's vital signs and talks to Paolo, (a doctor from the emergency territorial service), about the patient and her assessments. Paolo reports that he was unable to take any vascular access. He admits that he has no experience with pediatric patients and that in any case the patient was very vasoconstricted with cold ends and that was the reason why they were unable to achieve $\mathrm{O}_{2}$ saturation and that the HR was elevated $150 \mathrm{~min}$ despite a feverish temperature of $37.6{ }^{\circ} \mathrm{C}$. Paolo repeatedly apologizes for not having taken venous access, Mario listens absently to Paolo's indications on the patient and to his apologies, he is distracted by the appearance of the parameters on the monitor and by the difficulty in finding a saturation value.

Paolo knows he often faces difficulties with pediatric patients and this leads to further stress in their management.

\section{Critical Issues}

In this unstructured handover, Paolo forgets to report that he had performed a glycemic stick $(42 \mathrm{mg} / \mathrm{dl})$ which is also reported on the form filled up in the ambulance and left at the access to ER. At the triage, Giulia has inserted the patient into the waiting list through the ER software and she doesn't notice the filled format on the coun- ter. A structured handover, even in an emergency situation, can make a difference, especially in those situations where the team is aware of conditions that can generate stress, as in the case of Paolo that is unfamiliar with pediatric patients. The fact that Mario is distracted by the work of nurses in taking the parameters does not facilitate communication in such an important situation.

\subsubsection{9.20 pm}

The nurse Claudio began to place the electrodes on Anna's chest, and the oximeter probe on the index finger of the right hand.

- $\mathrm{HR}$ is $78 \mathrm{~min}$ down

- RR 16 min with gasping

- $\mathrm{satO}_{2}$ not detectable due to the presence of peripheral vasoconstriction

- T $37.4^{\circ} \mathrm{C}$

- BP 52-31 after some measurement attempts

Mario asked Sara to begin clinical evaluations and Lucia to take venous access.

\subsubsection{9.24 pm}

Paolo and Cesare have left the room and are preparing to go to the ambulance for another territorial service.

Anna does not even respond by now to the painful stimulus of venous access attempts.

In fact, in the meantime, Lucia has not been able to take the venous access, she has already been trying for $4 \mathrm{~min}$, on the patient's skin there are already signs of Paolo's ambulance attempts. Lucia knows she is very good at finding venous access in critically ill pediatric patients and does not accept failing in this case. She has lost track of time.

Sara has difficulty finding a peripheral pulse and assessing the patient's breathing. Mario sees Lucia's difficulties and begins to seek access to the veins of the hand.

The Claudio went to get a new oximeter probe. HR has fallen below $60 \mathrm{~min}$, a frequency that is now insufficient to maintain an effective cardiac output. 


\section{Critical Issues}

Anna's conditions because quickly compromised, but at the moment there is no real leadership, there is not an Event Manager who manages the situation. There is no explicit distribution of roles. Everyone is engaged in specific activities and even Mario, who initially seemed to assign the tasks, is busy with venous access. All the team is victim of an error of fixation: Mario and Lucia on the venous access, Claudio on the oximeter probe that seems not to work for evaluating the peripheral vasoconstriction, Sara in the search for the pulse and evaluation of the breathing in a clinical situation of imminent cardiac arrest. The perception of time and the possibility of a plan $B$ regarding venous access are also lacking. International guidelines show that in the critical child, if it is not possible to find venous access within $1 \mathrm{~min}$, it is a priority to insert an intraosseous device.

\subsubsection{9.27 pm}

- HR is 38 min down

- RR 6 min with gasping

- satO$_{2}$ undetectable

- BP undetectable

Claudio in attempts to have a parameter of saturation is repeatedly looking at the monitor and he noticed that the HR has dropped to $44 \mathrm{~min}$ and communicates it to the team in a generic way:

- "The heart rate is 44 min." Mario and Lucia are concentrated on the vascular access attempts and do not notice the communication, which is received by Sara. Sara, however, cannot understand if Mario's lack of response to this critical information is because he is distracted, but she does not stress the message because at that moment he has managed to cannulate a peripheral vein and they can start with taking the exams and starting a bolus of physiological solution at $20 \mathrm{ml} / \mathrm{kg}$. They don't know the weight, Claudio looks out of the door to ask the mother.

“11 kg, but how's Anna doing? What's up?"but the door has already closed the door.
Sara finally manages to attract Mario's attention with this sentence:

- "I don't feel the pulse, the heart rate is 34 on the monitor, she has gasping, we start cardiopulmonary resuscitation and we are calling the anesthesiologists to help us and intubate the patient."

\subsubsection{9.32 pm}

Beginning of CPR and contact the anesthesiologists.

As there is no real leadership, or an Event Manager who manages the patient with a $360^{\circ}$ view, communication skills has not been effective. The message is not addressed to a specific person. There was no feedback, no closing of the circle. Claudio's message on bradycardia did not reach Mario, the expert doctor and Sara, who got the message, does not want to interrupt Mario who is managing to cannulate a peripheral vein. Only later she kept Mario's attention by summarizing the clinical situation and proposing fundamental actions.

\subsubsection{9:34 pm}

CPR was on for 2 min when Martina, the anesthesiologist, and Giorgio, the nurse, arrive from the Intensive care.

She is informed that the patient is under cardiac arrest and that they have been massaging and ventilating her for about $2 \mathrm{~min}$.

Martina wants them to stop for $10 \mathrm{~s}$ to do a reassessment.

HR is 12 min electric activity without a pulse.

$\mathrm{RR}, \mathrm{satO}_{2}, \mathrm{PA}$ not detectable.

Martina decides to intubate the patient and gets help from Giorgio and asks Claudio.

"Meanwhile, get ready the adrenaline, $1 \mathrm{mg}$."

Claudio has some doubts about this drug, but prepares a $2.5 \mathrm{cc}$ syringe with $1 \mathrm{ml}$ of undiluted Adrenaline 1:1000, as he used to do in the geriatric ward, where he previously worked. Martina is on the third unsuccessful intubation attempt, and when she sees the syringe with undiluted adrenaline she is upset lashing out her frustration for the failed intubation on Claudio. 
"it is not possible! In pediatric arrest you always have to dilute 1 to 10,000 , always!!!" She takes the syringe and throws it in a corner of the room.

\section{Critical Issues}

Martina's request for adrenaline dosage is incorrect. Under the pressure of the moment she got confused between $\mathrm{mg}$ and $\mathrm{ml}$, asking a dose ten times higher than that required by guidelines. Besides the exchange related to the dosage, the rest of the communication on adrenaline was also incorrect, the dilution, the preparation method and Claudio's failure to explain the preparation explicitly led to a further waste of time. This technical error is frequent in pediatrics and numerous studies show that patients in this age group are exposed three times more than adults to potentially harmful therapeutic errors. In infants or low-weight patients, for example, there is a high risk of error: a minimal difference in the dosage of an additional drug can produce lethal effects since the low body mass and the immaturity of the organs cannot buffer the overdose. Martina's irritation related to the difficulties of intubation and the altered tone towards Claudio, definitively compromised a good team collaboration.

Luisa re-prepares the diluted adrenaline, but realizes that at the site of the cannulation of the vein there was an extravasation of Synovial Fluid and the vein is irrecoverable. She tells Mario that is massaging the patient, he tries to see if the Synovial rilli sis really leaking, but this worsens the quality of the massage.

Martina listens to the conversation and decides for an intraosseous access and sends Giorgio to take the drill in the intensive care unit. The same intraosseous is present in the ER, but at this point Sara and Claudio, who know about that, don't want to contradict Martina. Mario thinks that in the intensive care unit there could be a more advanced technological device to do this procedure, but does not have the courage to ask for it.

\section{Critical Issues}

The tense atmosphere within the team again leads to unshared choices, and the lack of information (the presence of the ER intraosseous drill) with the consequent further loss of time and resources (Giorgio leaves the room to go to intensive care unit). ER and intensive care unit teams were taking care of the same patient without speaking enough and without sharing priorities.

Anna is in asystole.

After about 40 minutes CPR without ever resuming a pulse compatible rhythm, Martina declares Anna's death. And now someone has to open that door to communicate it to the mother.

\subsubsection{Final Considerations}

Pediatrics is one of the most challenging areas in patient safety. There is a need for basic and advanced research and for a rigorous application of the evidence-based solutions already tested and validated in pediatric settings. The application of these solutions remains a challenging issue. The clinical case we presented and the previous evidences discussed in the chapter underlines the importance of understanding the context as a key factor for safety, and especially for safety in pediatrics. The importance of understanding context has been highlighted by research underlining that proven safety solutions can be less effective in settings different from the ones they were developed and firstly applied in [27]. This opens a large debate about the effectiveness of the proposed interventions but also on the capability of the new contexts, in terms of organizational, cultural, and economic resources, to apply that solution [ibidem]. In this scenario we believe that quality improvement methodology together with the human factor approach can provide fundamental insight for the transferability and success of the application of the patient safety solutions in different contexts and especially in pediatrics. Among the key elements responsible for the comprehension and the enabling of the context are:

- Patient and family engagement, which has been identified as a priority in the WHO patient safety Declaration during WHA 2019 [28] and is one of the most important elements for setting up agendas at all levels for promoting patient safety.

- Leadership and culture which are the engines for fostering patient safety as a strategic asset 
of healthcare organizations, usually more developed and advanced in pediatrics.

- Governance, which is the basic framework in which developing patient safety actions.

- Teamwork and education, which are the infrastructure for creating new generations of clinicians able to provide care having in mind safety as a priority for our kids.

\section{References}

1. Walsh KE, Bundy DG, Landrigan CP. Preventing health care-associated harm in children. JAMA. 2014;311(17):1731-2. https://doi.org/10.1001/ jama.2014.2038.

2. Mueller BU, Daniel RN, Stucky Fisher ER, Council on Quality Improvement and Patient Safety, Committee on Hospital Care. Principles of pediatric patient safety: reducing harm due to medical care. Pediatrics. 2019;143(2):e20183649. https://doi.org/10.1542/ peds.2018-3649.

3. The WHO's Global Patient Safety Challenge: Medication without harm. May 2017. WHO/HIS/ SDS/2017.

4. Santell JP, Hicks R. Medication errors involving pediatric patients. Jt Comm J Qual Patient Saf. 2005;31(6):348-53.

5. Kaushal R, Bates DW, Landrigan C, et al. Medication errors and adverse drug events in pediatric inpatients. JAMA. 2001;285(16):2114-20.

6. Matlow AG, Baker GR, Flintoft V, et al. Adverse events among children in Canadian hospitals: the Canadian Paediatric Adverse Events Study. CMAJ. 2012;184(13):E709-E7181.

7. Khan A, Furtak SL, Melvin P, Rogers JE, Schuster MA, Landrigan CP. Parent reported errors and adverse events in hospitalized children. JAMA Pediatr. 2016;170(4):e154608.

8. Mohr Julie J, Carole ML, Thoma Kathleen A, Woods $\mathrm{D}$, et al. Learning from errors in ambulatory pediatrics. In: Henriksen K, Battles JB, Marks ES, et al., editors. Advances in patient safety: from research to implementation, Research findings, vol. 1. Agency for Healthcare Research and Quality: Rockville, MD; 2005.

9. Stang A. Safe care for pediatric patients: a coping review across multiple health care settings. Clin Pediatr. 2018;57(1):62-7.

10. Reason J. Human error. 1st ed. Cambridge: Cambridge University Press; 1990.

11. Barach P, Small SD. Reporting and preventing medical mishaps: lessons from non-medical near miss reporting systems. BMJ. 2000;320:759-63.

12. Woods D, Thomas E, Holl J, Altman S, Brennan T. Adverse events and preventable adverse events in children. Pediatrics. 2005;115(1):155-60.

13. Thomas EJ, Brennan TA. Errors and adverse events in medicine: an overview. In: Vincent CA, editor.
Clinical risk management. Enhancing patient safety. London: BMJ Publications; 2001.

14. Carayon P, Schoofs Hundt A, Karsh B, et al. Work system design for patient safety: the SEIPS model. BMJ Qual Saf. 2006;15:i50-8.

15. Seymour NE, Gallagher AG, Roman SA, O'Brien MK, Bansal VK, Andersen DK, et al. Virtual reality training improves operating room performance: results of a randomized, double-blinded study. Ann Surg. 2002;236(4):458-63.

16. Sarker SK, Chang A, Vincent C, Darzi AW. Surgical technical errors in performing open \& laparoscopic surgery. Br J Surg. 2004;91:s78.

17. Sarker SK. Courses, counsellors \& cadavers: reducing errors in the operating theatre. BMJ. 2003;327:s10.

18. Hadjianastassiou VG, Tekkis PP, Poloniecki JD, Gavalas MC, Goldhill DR. Surgical mortality score: risk management tool for auditing surgical performance. World J Surg. 2004;28(2):193-200.

19. Birkmeyer JD, Siewers AE, Finlayson EVA, Stukel TA, Lucas FL, Batista I, et al. Hospital volume and surgical mortality in the United States. N Engl J Med. 2002;346:1128-37.

20. Begg CB, Riedel ER, Bach PB, Kattan MW, Schrag $\mathrm{D}$, Warren JL, et al. Variations in morbidity after radical prostatectomy. N Engl J Med. 2002;346:1138-44.

21. World Health Organization. Patient safety curriculum guide. Available from: http://www.who.int/ patientsafety/education/curriculum/tools-download/ en/.

22. Rasmussen J. Skills, rules, knowledge: signals, signs and symbols and other distinctions in human performance models. IEEE Trans Syst, Man, Cybern, SMC13. 1983. p. 257-67.

23. Fitzsimons J, Vaughan D. Top 10 interventions in paediatric patient safety. Curr Treat Options Peds. 2015;1:275-85. https://doi.org/10.1007/ s40746-015-0035-3.

24. Shekelle PG, Wachter RM, Pronovost PJ, et al. Making health care safer II: an updated critical analysis of the evidence for patient safety practices. Evid Rep Technol Assess. 2013;211:1-945.

25. Mirza A, Winer J, Garber M, Makker K, Maraqa N. Primer in patient safety concepts: simulation case-based training for pediatric residents and fellows. MedEdPORTAL. 2018;14:10711. https://doi. org/10.15766/mep_2374-8265.10711.

26. Institute of Medicine (US) Committee on Quality of Health Care in America. In: Kohn LT, Corrigan JM, Donaldson MS, editors. To err is human: building a safer health system. Washington, DC: National Academies Press; 2000.

27. Dixon-Woods M, Leslie M, Tarrant C, Bion J. Explaining Matching Michigan: an ethnographic study of a patient safety program. Implement Sci. 2013;8:70.

28. Patient safety resolution, Global action on patient safety. Reported by General Director, Seventy-Second World Health Assembly A72/26, 25 Mar 2019. http://apps.who.int/gb/ebwha/pdf_files/WHA72/ A72_26-en.pdf. Accessed 04 Jan 2020. 
Open Access This chapter is licensed under the terms of the Creative Commons Attribution 4.0 International License (http://creativecommons.org/licenses/by/4.0/), which permits use, sharing, adaptation, distribution and reproduction in any medium or format, as long as you give appropriate credit to the original author(s) and the source, provide a link to the Creative Commons license and indicate if changes were made.

The images or other third party material in this chapter are included in the chapter's Creative Commons license, unless indicated otherwise in a credit line to the material. If material is not included in the chapter's Creative Commons license and your intended use is not permitted by statutory regulation or exceeds the permitted use, you will need to obtain permission directly from the copyright holder. 